\title{
Sensory interactions in bilateral kinesthesia
}

\author{
ROBERTA D. ROBERTS AND GLYN W. HUMPHREYS \\ University of Birmingham, Birmingham, England
}

\begin{abstract}
Three experiments are reported that examine the masking effect on the detection of kinesthetic targets from noise presented simultaneously on the same hand or on a different hand as the target. Performance was facilitated when noise on the two hands was correlated versus uncorrelated or when it occurred on just one hand. The data are consistent with correlated input on the two hands being compared and used to reduce noise effects. Moreover, participants ignored uncorrelated noise from nontarget hands when they knew in which hand the targets would occur. There was no effect of shifts from uncorrelated to correlated noise $200 \mathrm{msec}$ prior to targets, suggesting that such bottom-up changes were insufficient to induce comparison across the hands. In contrast, shifts from correlated to uncorrelated noise $200 \mathrm{msec}$ prior to the target had performance at the same level as that with uncorrelated noise throughout the trial. This result indicates that the loss of correlation was detected within this interval and that when the target's location was known, participants were then able to switch attention to the target hand. The interactions between attentional filtering and the correlation on the processing of bilateral kinesthetic inputs are discussed.
\end{abstract}

Many of our interactions with our environment are accomplished with the use of both of our hands. Such use can involve different movements of the two hands to achieve a common goal, such as one hand stabilizing and turning an object while the other hand explores the object's surfaces (Lederman \& Klatzky, 1987). It can also involve actions in which the movements of the hands are very similar; for instance, when judging the weight of a large object, we might lift and heft it with both hands. The coupled use of the arms is often studied in the context of movement control - that is, how we behave when we have to produce movements. A common finding is that control of the arms appears to be shared, or under a single command, when both arms are being used together. For instance, research has shown that although the movement of a single arm between two targets on a table is well described by Fitts's law in terms of its speed-accuracy relationships (Fitts, 1992), this law no longer applies under bimanual conditions. When the arms are moved simultaneously to targets of different distances, the movements are coordinated in time so that the hands arrive at their respective targets together (Kelso, Southard, \& Goodman, 1979). This coupling across the hands persists even when an obstruction is placed in the path of one hand. The movement of the unobstructed hand is not only temporally coupled to that of the obstructed one, but it also shows a similar deviation in space, although with a lower amplitude (Kelso, Putnam, \& Goodman, 1983). Coupling between the hands is not restricted to these relatively simple pointing situations, but is also found under conditions in which each hand may have a different functional task (e.g., one hand opening a drawer while the other reaches into it; Perrig, Kazennikov, \& Wiesendanger, 1999), as well as in studies of bimanual force control (Serrien \& Wiesendan- ger, 2001a, 2001b). Researchers have suggested that such temporal and spatial coupling between the hands facilitates movement control by simplifying the organization needed to control two tasks to that of controlling just a single unit (Kelso et al., 1979).

Although the production of movement has an important role in creating this coordination, note that the sensations arising from these bilateral movements will, as a result, be coordinated in their spatiotemporal properties. One must ask whether the central nervous system might make use of this sensory consequence of the coupling system. That is, when we are presented with sensory inputs that are correlated in their changes over time, is there an assumption of a common cause? Does correlation in sensations across the hands change the way in which we process the sensations on each hand?

There are few studies of sensitivity to correlation in the sensations received from the hands. We are aware of only three such studies to date (Kitada, Kochiyama, Hashimoto, Naito, \& Matsumura, 2003; Roberts, Humphreys, \& Wing, 2005; Wilson, Bingham, \& Craig, 2003). The ability to judge whether one or two separate objects rolled across the surface of two fingers has been found to vary as a function of the relative phase of the stimulation (Kitada et al., 2003). A single cylinder rolled across the fingers with an in-phase pattern was more likely to generate a "one-stimulus" judgment than when the same cylinder was rolled across both fingers with some rotation so that the fingers were stimulated in an antiphase pattern. The perception of a single stimulus was unaffected by whether it was one or two cylinders rolling across the fingers, as long as the stimulation was in-phase. When the antiphase movements across the fingers were generated by two separate cylinders, participants were

R. D. Roberts, r.roberts@bham.ac.uk 
highly unlikely to report a single object. This difference in sensation when using one as compared with two cylinders is likely to have been due to higher, albeit negative, correlations in the one-cylinder condition than in the two-cylinder condition. The overall pattern of results suggests that the level of correspondence between multiple touch inputs affects the way in which we interpret these stimuli.

Note that the above results were found for the tactile stimulation of two fingers on a single hand. However, relative phase has also been shown to have a role in the way kinesthetic inputs from both hands are processed. For example, Wilson et al. (2003) demonstrated that we are more sensitive to some rhythmic patterns between our limbs than to others. Sensitivity to relative phase between the hands was investigated by asking participants to track the movements of a set of levers with their left and right index fingers. On each trial, the levers moved through $8 \mathrm{sec}$ of a sinusoidal pattern, with the mean relative phase between the fingers of each hand being $0^{\circ}, 90^{\circ}$ or $180^{\circ}$. Sensitivity to correspondence between the hands was measured by introducing variability to the mean relative phase and asking participants to rate the level of this variability. The better that participants are able to encode mean relative phase, the easier it will be to discriminate deviations from this phase relation (Zaal, Bingham, \& Schmidt, 2000). Research found that variability discrimination was best when the movement of the two fingers was in-phase. Performance declined with a $180^{\circ}$ relative phase difference between the fingers, but it was worst when relative phase was $90^{\circ}$. In addition, increases in frequency had little effect on perception in the $0^{\circ}$ condition, but they led to increases in judgments of phase variability when the mean relative phase was $180^{\circ}$.

These data suggest that people are most sensitive to the phase relations that they are best able to produce. Rhythmic movements of the two hands in untrained participants are most stable when they are in-phase, less stable when they are in antiphase (i.e., with a $180^{\circ}$ phase lag between the hands), and unstable (show greater variability) at other phase relations (Kelso, 1984; Yamanishi, Kawato, \& Suzuki, 1980). In-phase coordination of the limbs is unaffected by increases in the frequency of the movement. In contrast, antiphase movements become less stable with increases in frequency, and under laissez-faire instructions, antiphase movements switch to become in-phase. Thus, it seems that sensitivity to phase relations across passively displaced hands mirrors findings from situations in which participants have to move their own hands.

Perceptual sensitivity to relative phase has also been demonstrated for visual stimuli. In a set of studies, Bingham and colleagues (Bingham, Schmidt, \& Zaal, 1999; Bingham, Zaal, Shull, \& Collins, 2001; Zaal et al., 2000) carried out similar experiments using visual rather than kinesthetic stimuli. Participants rated the stability of the relative phase of two visual oscillators whose motion was either based on actual human movement (Bingham et al., 1999) or was computer generated (Bingham et al., 2001; Zaal et al., 2000). The results mirrored those discussed previously. In all cases, the movements were judged as being most stable when the mean relative phase was $0^{\circ}$ and, to a lesser extent, $180^{\circ}$. Participants found it difficult to judge the level of variability at other phase relations, even reporting high levels of variability when the relative phase of the stimuli was invariant (Zaal et al., 2000). As was expected, judgments of visual phase variability were affected by frequency when the mean relative phase was $180^{\circ}$ but not when it was $0^{\circ}$.

The results for kinesthetic stimuli suggest that people are able to encode sensory correspondence across the hands; however, in all the testing situations used hitherto, participants were asked to focus on and report the bilateral relationship. Using a more indirect approach in which the coupling between the hands was not the main focus of the task, we (Roberts et al., 2005) were able to demonstrate that relationships across the hands can still affect perception. In our study, participants were asked to localize a target movement pattern in kinesthetic noise. The noise consisted of a sequence of random movements of the index fingers. The noise on the left and right index fingers could be positively correlated, negatively correlated, or uncorrelated. Participants were asked to judge whether a short "up and down" ramp movement (the target) occurred on the left or the right index finger. Target and noise movements were applied to passive observers (reducing the possibility that perceptual judgments were based on anything other than afferent information). Target localization was better in positively correlated noise than in negatively or uncorrelated noise, whereas performance did not differ in the negatively and uncorrelated conditions. Interestingly, there was some dissociation between the ability to explicitly detect correlations between the hands and the effect that such correlations had on perception. Although the advantage for target discrimination with positive correlation persisted with a phase lag of $200 \mathrm{msec}$, explicit discrimination of correlation was at chance when the noise was no longer in phase.

These results suggest that particular forms of correspondence across our hands can have an important role in shaping our perception, and that the effects are apparent even when the manipulation between the stimuli is implicit and cannot be explicitly reported. This does not mean, however, that attention has no influence on our sensitivity to correlation between kinesthetic inputs. The effects of attention were investigated in the present article.

There is some evidence that stimulation of a taskirrelevant — and therefore, likely unattended — limb affects the active control of a contralateral limb. The coupling between a passively moved but task-irrelevant hand and an actively controlled moving hand has been shown to affect the ability to maintain rhythmic movement between an active hand and both an active leg (Serrien, Li, Steyvers, Debaere, \& Swinnen, 2001) and an external, auditory metronome (Ridderikhoff, Peper, \& Beek, 2005, 2006). Inphase and antiphase movements of a homolateral arm and leg are less stable when the other arm or leg is passively moved. These effects are strongest when the active limbs are moved in antiphase (Serrien et al., 2001). Movements of the active hand in time to a metronome are also affected by movements that are imposed on the passive hand but are phase shifted relative to the metronome (Ridderikhoff et al., 2005, 2006). It is possible that the inputs from the unattended, passively moved limb automatically draw attention and thus affect performance. On the other hand, 
some have suggested that these coupling effects might have a spinal origin (Ridderikhoff et al., 2005). In either case, impairments to performance caused by the inputs from the task-irrelevant limb cannot be excluded.

Extending these findings from movement control to perception, we investigated whether the effects of bilateral correspondence on perceptual tasks persist when one hand is no longer task relevant. This question was addressed in the present set of experiments using the paradigm from our previous study (Roberts et al., 2005). We examined whether correlation across the hands affected target detection when there was no longer a requirement to process the noise stimuli of both hands. Task relevance was manipulated by providing cues to the location of targets that were embedded in kinesthetic noise with various bilateral relationships.

\section{GENERAL METHOD}

\section{Equipment}

Two linear motors (actuators; Digifade 1000 linear drives) were used to move the participant's index fingers up or down by flexion and extension about the MCP joint (see Figure 1). The contact surface of each actuator was slightly domed, smooth metal with a diameter of $23 \mathrm{~mm}$. There was $30 \mathrm{~mm}$ of spacing between actuator centers. During the experiment, participants were seated in front of the actuators with their forearms resting on a table surface. They were asked to rest each index finger pad lightly on one of the two actuators. The actuators held the fingers $18 \mathrm{~mm}$ above the surface of the table. The rest of the hand remained flat on the table. The participants' fingers were secured to the actuators with a pliable fabric loop over the top of the fingers in order to keep the finger pad in contact with the smooth actuator surface. Participants lifted their toe or heel off of a set of foot switches in order to indicate decisions about target signals.

Participants were asked to remain passive while their fingers were displaced. This contrasts with the studies of Wilson et al. (2003) in which participants actively tracked the movements of the manipulanda. Our aim was to study the interaction between the two sensory channels in the absence of any other predictive mechanisms. To this end, we used pseudorandom patterns of movements rather than the more predictable sinusoidal movements that were used by Wilson and colleagues. We anticipated that participants would experience more difficulty in tracking pseudorandom movements. In addition, an active tracking paradigm would make the noise stimuli the focus of the task. When one hand was no longer task relevant, participants might either experience difficulty tracking the movement on the nontarget hand or actively track the movements, which may change the way in which attention is distributed across the hands. To avoid these issues, kinesthetic stimuli were applied to passive observers.

\section{Noise Waveforms}

In each experiment, a base set of noise waveforms was generated in advance of data collection by a random selection of a series of successive actuator positions in the range $0-10 \mathrm{~mm}$. Before each waveform was accepted in the base set, a check was made in order to ensure that the lag-zero cross-correlation between noise waveforms for the two hands was less than ( \pm .5$)$. If this was not the case, then a new noise waveform was generated and checked against the cross-correlation criterion. A different base set of 20 waveforms was produced for each of the experiments. During each trial, the noise waveform was output by selecting the next end position in succession every $200 \mathrm{msec}$. Drive software, matched to the inertial and drag characteristics of the actuators, generated a half-sinusoid positiontime function so that the actuator moved smoothly between the start and target position. The average frequency spectrum of the resulting motion of the actuator was uniform in the range of $1-6 \mathrm{~Hz}$, with smooth decrease below and above this range to zero power at 0 and $10 \mathrm{~Hz}$. The autocorrelation function on average decreased smoothly to 0 at lags of $200 \mathrm{msec}$ and beyond, with a range of -.5 to +.5 . The cross-correlation between the set of all basic noise waveforms was, on average, 0 across all time lags, with a range of -.5 to +.5 .

\section{Procedure}

Each trial lasted $4 \mathrm{sec}$, during which time the two actuators moved each index finger with a noise waveform that was selected from the base set. A target movement was either present or absent. In all of the experiments, the target peak amplitude was fixed at $6 \mathrm{~mm}$. This value is the mean of thresholds found to give $79 \%$ correct performance in previous localization experiments (Roberts et al., 2005). These threshold values were found using adaptive procedures. The standard error of

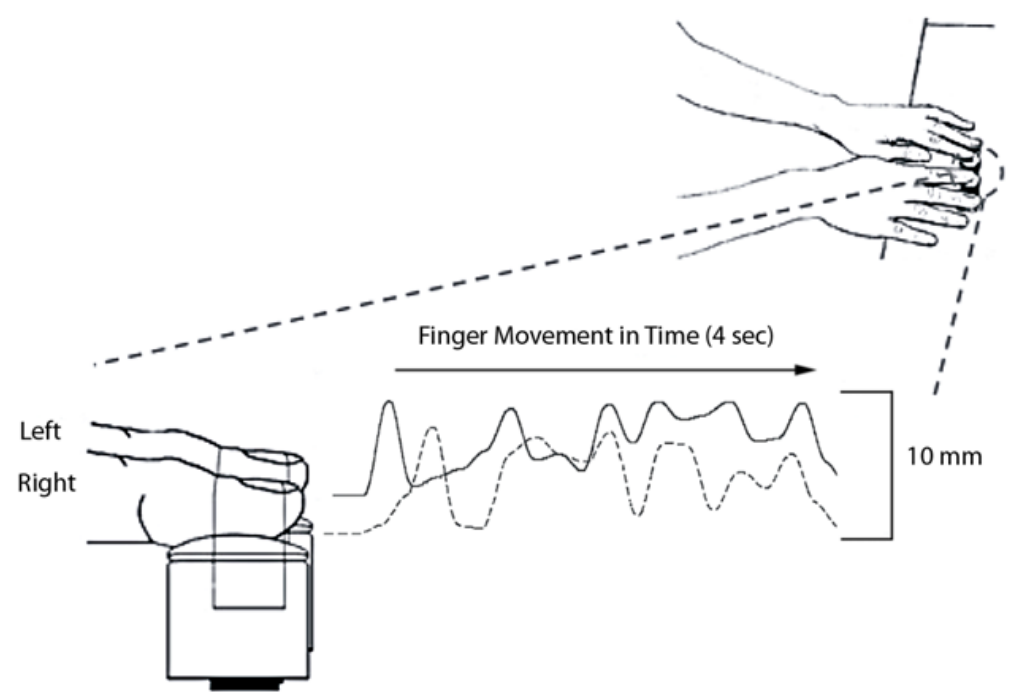

Figure 1. Experimental setup. The upper part of the figure shows the arrangement of the hands. The lower part shows the left and right index fingers resting on a set of actuators used to move the fingers. The movements of the right finger are shown by the dashed line, those of the left finger by the solid line. 
this mean was $0.42 \mathrm{~mm}$. Pilot testing for the present set of experiments showed that this threshold value generated performance above chance in the uncorrelated condition. The trials were presented in blocks with equal numbers of target-present and target-absent trials in a block. The order of target-present and target-absent trials was randomly determined. Targets were 1-sec long smoothed ramps with quartered-sine onset and offset and were added to the noise waveform after $2 \mathrm{sec}$ of motion. At the start of each block of trials, participants were directed to expect the target either at a specific hand (when the target location was blocked and therefore certain) or at either hand (when the target location was uncertain). In this latter case, on half of the target-present trials, the target was on the left finger, with the remainder on the right and the order randomly determined. In either case, at the end of a trial, participants lifted either their right toe or right heel from a foot pedal to indicate whether the target was present or absent. The participants kept their eyes closed throughout the testing. Auditory feedback was provided as to whether the response was correct.

Each trial, including time taken to make a response, lasted around $6 \mathrm{sec}$. The experiments were run using a single interval yes-no procedure. Differences in the proportion of correct responses in single interval tasks may reflect both changes in sensitivity as well as different tendencies to give one response or another. Thus, signal detection theory (SDT) measures of sensitivity $\left(d^{\prime}\right)$ and criterion (c) (Macmillan \& Creelman, 1991) were used to provide an index of the participants' ability to detect targets separately from their bias toward reporting the target as being either present or absent. The $d^{\prime}$ values were converted into proportion-correct values.

\section{EXPERIMENT 1}

We examined whether there was any difference in the detection of a kinesthetic ramp on one finger when it was masked by perfectly correlated, bilateral, kinesthetic noise as compared with kinesthetic noise that was uncorrelated across the fingers. Detection in these bilateral conditions was compared with measures that were taken when the noise was on one hand alone. We also examined whether the effects of correspondence in bilateral noise were sensitive to the task relevance of both hands. The relevance of the inputs from the two hands was manipulated by providing participants with differing information about the location of the target. For the group of participants with knowledge of the target location, only the finger potentially containing the target was ostensibly relevant for completing the task. However, for the group without information about the location of the target, the inputs from both fingers had to be monitored for the target. Both groups of participants were given no instructions about the correlation in the noise signals.

\section{Method}

Participants. Thirty participants, 24 female and 6 male, took part in this experiment after giving informed consent. Their ages ranged from 19 to 37 years $(M=22, S D= \pm 4.1)$. Twenty-seven participants were right handed, 3 were left handed for handwriting, and all were paid for the time spent in the experiment. These participants were not informed of the purpose of this study.

Procedure. Participants were presented with 4-sec-long noise waveforms on either their left and right index fingers or one finger alone. On half of the trials, a 1-sec smoothed ramp target was added to the noise waveform of one hand (the target hand). All participants were told that the target had an equal probability of being present or absent. For half of the participants, the target hand would remain the same for an entire block of trials, with the target appearing on one hand during the first block and then switching to the other hand during the second block. For the remaining 15 participants, the target had an equal chance of appearing on the left hand as it did on the right. In either case, participants had to decide whether a target was present or absent. Target-present responses were made by lifting a foot pedal under the toe of the right foot, and target-absent responses were made by lifting a foot pedal under the right heel.

Target location known. Participants who were tested in the conditions in which the target location was known experienced a total of 180 trials that were run in two blocks consisting of 90 trials each. In one block, the target would only occur on the left; in the other, the target would only occur on the right. At the start of each block of trials, participants were told on which hand the target would appear. The target location always coincided with the moving hand in the onehand condition; thus, targets were always presented in noise. In both blocks, half of the trials contained targets and the other half did not. The trials for the different noise (positively correlated, uncorrelated, and unilateral) conditions were interleaved within each block.

Target location unknown. When the target location was unknown, each participant was tested on a total of 240 trials that were run in four blocks consisting of 60 trials each. On each trial, the target could occur on either the left or the right finger. In the unilateral condition, this meant that half of the targets were presented on a finger that was, apart from the motion created by the target, stationary. In all of these blocks, half of the trials contained targets, and the other half did not. The trials for the different noise conditions (positively correlated, uncorrelated, and unilateral) were interleaved within each block.

\section{Results}

The mean proportion correct values for Experiment 1 are shown in Figure 2. The data for the two groups of participants were analyzed independently for effects of stimulus presentation order. Since neither group showed any effects of stimulus order or interactions of stimulus order with other factors, this factor was not included in subsequent analyses. The data were analyzed using a mixed-design ANOVA, with the within-subjects factor noise (positively correlated, uncorrelated, and unilateral) and the betweensubjects factor target location (known and unknown).

There was a reliable effect of noise $[F(2,56)=6.955$, $p<.01]$, but no main effect of target location $[F(2,28)=$ $1.069, p=.310]$, and no significant interaction between the two factors $[F(2,56)=0.881, p=.420]$. Individual comparisons revealed that target detection in positively correlated noise was better than when there was uncorrelated noise $[t(29)=3.570, p<.01]$ and when there was noise on one hand $[t(29)=-2.872, p<.01]$. In contrast, there was no difference between target detection in un-

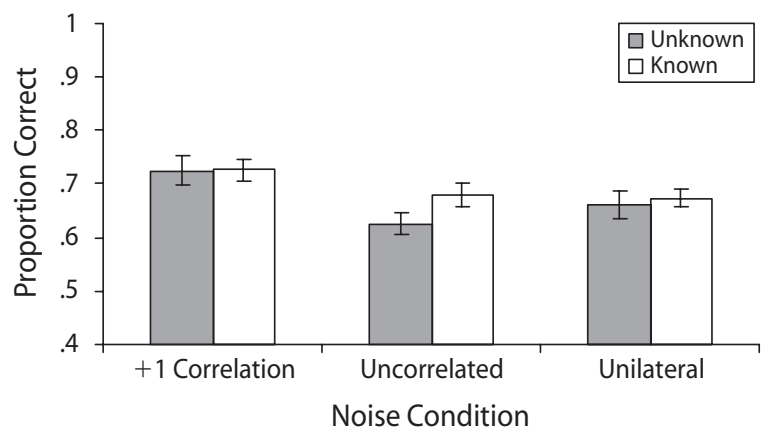

Figure 2. Data from Experiment 1 showing bias-free proportion correct in the target detection task with positively correlated, uncorrelated, and unilateral noise conditions. Error bars show one standard error. 
correlated noise and unilateral noise $[t(29)=0.685, p=$ .499]. Knowledge of target location had no effect when the noise was on one hand alone $[t(28)=0.404, p=.689]$ or was correlated $[t(28)=-0.031, p=.976]$. However, the effect of knowledge of the target location on performance approached significance when the noise was uncorrelated $[t(28)=1.762, p=.089$; two-tailed $]$.

It was not possible to derive a bias-free sensitivity value for the unilateral conditions when the target was embedded in noise and when it occurred on a motionless finger, since there was no way to decide which of the target-absent trials belonged to each condition. However, it is conceivable that it was easier to detect targets that occurred on the index finger without the noise waveforms. This possibility is supported by the proportion-correct data, which showed that there was detection of a larger number of targets when they were on the motionless finger than when they were embedded in the finger with the noise waveform $[t(14)=$ $-1.515, p=.038$; one-tailed] (unilateral targets in noise, $M=0.53, S D= \pm 0.08$; unilateral targets without noise, $M=0.67, S D= \pm 0.03)$.

The criterion data are shown in Figure 3. These data were analyzed using a mixed-design ANOVA, with noise (positively correlated, uncorrelated, and unilateral) as the within-subjects factor and target location (known and unknown) as the between-subjects factor. There was an overall effect of noise on the criterion shown by participants $[F(2,56)=18.524, p<.01]$. There was no main effect of target location on criterion $[F(1,28)=0.002, p=.965]$. The interaction between noise and target location was significant $[F(1,28)=9.470, p<.01]$.

There was a significant overall tendency to produce target-absent responses when targets were embedded in positively correlated noise $[t(29)=6.867, p<.01]$. This bias was unchanged by knowledge of the target's location $[t(28)=-.827, p=.415]$. With uncorrelated noise, the criterion did not differ from zero when target location was unknown $[t(14)=-1.349, p=.199]$; however, there was a bias for target-absent responses when the target location was known $[t(14)=4.169, p<.01]$. This difference was statistically significant $[t(28)=-3.749, p<.01]$.

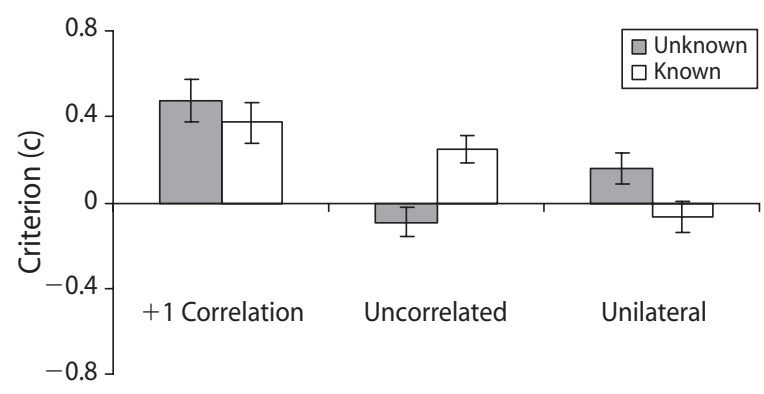

Noise Condition

Figure 3. Data from Experiment 1 showing averaged criterion (c) in the target-detection task with positively correlated, uncorrelated, and unilateral noise conditions. Negative values indicate a bias for "target-present" responses, and positive values indicate a bias for "target-absent" responses. One standard error is shown.
The reverse pattern of results occurred for participants' response criteria when noise occurred on just one hand. The bias for target-present responses emerged when the target location was unknown $[t(14)=2.182, p<.05]$, but there was no bias when the target location was known $[t(14)=-1.145, p=.271]$. This difference in criterion was reliable $[t(28)=2.406, p<.05]$.

\section{Discussion}

There was a marked effect of correlation of noise on target detection. Proportionately, more targets were detected when they were embedded in positively correlated noise waveforms than when they were embedded in uncorrelated noise waveforms. This effect is consistent with previous findings (Roberts et al., 2005). Interestingly, positive correlation in bilateral noise waveforms led to better target detection than in unilateral noise waveforms. Overall, there was no difference in performance when the noise was uncorrelated than when it was unilateral. Furthermore, knowledge of the location of the target had no reliable effect on performance in any of the noise conditions.

These findings indicate that the ability to separate a target from noise was improved with an extra channel of noise input, but only when the two channels of noise were identical. This improvement resulting from a bilateral pattern remained under conditions in which one hand was no longer task relevant. That is, even when the target's location was known, people continued to process the inputs from both hands rather than filtering out the inputs from the nontarget hand.

Unexpectedly, having two sources of uncorrelated noise did not lead to worse performance than when the target was embedded in unilateral noise. When the target location was known, only the hand potentially containing the target was relevant for target detection. It follows that if, in the uncorrelated condition, noise from the nontarget hand was filtered out, performance should be no different from that with noise on one hand alone. This pattern was found in the data. Interpretation of the data when the target location was unknown is less obvious. Although there was a numerical change with knowledge of target location in the uncorrelated condition, this difference was not reliable. Performance in the unilateral condition showed no change with knowledge of target location. However, note that performance in this latter condition reflects the detection of targets embedded in a noise waveform, as well as those occurring on an otherwise motionless finger. This manipulation was used in order to ensure that participants were dividing their attention between both fingers. Examining the noise and no-noise unimanual conditions in isolation showed that performance was better when the target was on a motionless finger than when it was embedded in noise. When the target occurred in noise and its location was unknown, performance was at chance levels. This result contrasts with performance in similar noise conditions when the target location was known, suggesting a cost to attending to more than one location for a target. However, these conclusions should be tentative, since the values in the unilateral, unknown condition cannot be adjusted for bias and thus cannot be directly compared with those in the other conditions. 
The participants in this experiment also showed reliable preferences for one response over another. Those with knowledge of the target's location favored target-absent responses in both the positively correlated and uncorrelated noise conditions, but showed no preference in the unilateral noise condition. Participants without knowledge of target location showed a preference for targetabsent responses in the positively correlated and unilateral conditions, but no bias in the uncorrelated condition. It is possible that these patterns of bias are particular to the two groups and that changes in criterion with target location simply reflect the use of a between-subjects design. This possibility was examined in Experiments 2 and 3, in which a within-subjects design was used.

Overall, the results of Experiment 1 provide little evidence of the filtering of sensory inputs from a taskirrelevant hand. There was no evidence of filtering when the noise waveforms were positively correlated and performance could be improved by attending to the inputs from both hands. However, it was also the case when the noise inputs were either uncorrelated or unilateral. We examined this lack of filtering in two further experiments by changing the correlation in bilateral noise in the time window in which a target could occur and observing any effects of knowledge of target location on performance.

\section{EXPERIMENT 2}

Experiment 2 examined the possibility that the processes that are taking place and giving rise to improved target detection in the positively correlated condition also occur when the noise is uncorrelated. To assess this possibility, we compared performance with uncorrelated noise throughout the presentation (as in Experiment 1) with conditions in which a brief period of correlated noise was added, prior to the target occurring ("drop-in" trials). If performance in the positively correlated and uncorrelated conditions makes use of the same processes, then there should be no filtering of the information on the nontarget hand when the target's location is known, and this should hold for both conditions. Moreover, target detection should improve with a short burst of correlated noise during the period in which a target may occur.

The first $1,800 \mathrm{msec}$ of a drop-in trial were the same as those of an uncorrelated trial; the noise waveforms on the left and right fingers had a very low degree of correlation. However, $200 \mathrm{msec}$ before and up to $200 \mathrm{msec}$ after the period in which the target could occur, the noise movements of the fingers became positively correlated. The waveform on one of the two fingers (selected at random) was replaced by a copy of the waveform on the other hand. Thus, for a period of 1,400 msec (during which the 1-sec target could occur), the noise movements of the two hands were positively correlated; then, $200 \mathrm{msec}$ after the target, the movements of the index fingers once again became uncorrelated.

\section{Method}

Participants. Eighteen participants, 14 female and 4 male, participated in this experiment after giving informed consent. Their ages ranged from 19 to 45 years $(M=24, S D= \pm 6.3)$. Sixteen par- ticipants were right handed, 2 were left handed for handwriting, and all were paid for the time spent in the experiment. These participants were not informed of the purpose of this study and had not taken part in the previous experiment.

Procedure. The procedure was the same as that used in Experiment 1.

Design. The noise waveforms could be positively correlated throughout, uncorrelated throughout, or uncorrelated with a short burst of positively correlated noise during the target period (the drop-in condition). The participants were not informed of the different noise conditions.

Each participant was tested on a total of 360 trials that were run in three blocks of 90,90 , and 180 trials. In one block of 90 trials, the target would only occur on the left; in the other block of 90 trials, the target would only occur on the right. In both of these blocks, participants were told in advance on which hand the target would appear (target-known condition). In contrast, targets occurring during the block of 180 trials could appear on the left or right hand, and participants had no prior knowledge of target position (target-unknown condition). For all blocks, half of the trials contained targets, and the other half did not. The order of the location conditions (known vs. unknown) was balanced across the participants. The trials for the different noise conditions were randomly interleaved within each block.

\section{Results}

The proportion of correct responses averaged over participants is shown in Figure 4. There was no main effect of the order in which the different location conditions (known vs. unknown) were run. In addition, there were no reliable interactions involving location order. Consequently, all of the following analyses reported were averaged across order. The data were analyzed using a within-subjects ANOVA with target location (known and unknown) and noise (positively correlated, uncorrelated, and drop-in) as factors.

There was a reliable main effect of noise $[F(2,34)=$ $15.555, p<.01]$ and of knowledge of target location $[F(1,17)=9.571, p<.01]$ on performance. The interaction between noise and target location was not significant $[F(2,34)=0.282, p=.721]$.

Target detection was better when the noise was positively correlated than when it was uncorrelated $[t(17)=$ $3.961, p<.01]$, and performance with correlated noise was better than that in the drop-in condition $[t(17)=$ $5.375, p<.01]$. There was no difference in performance

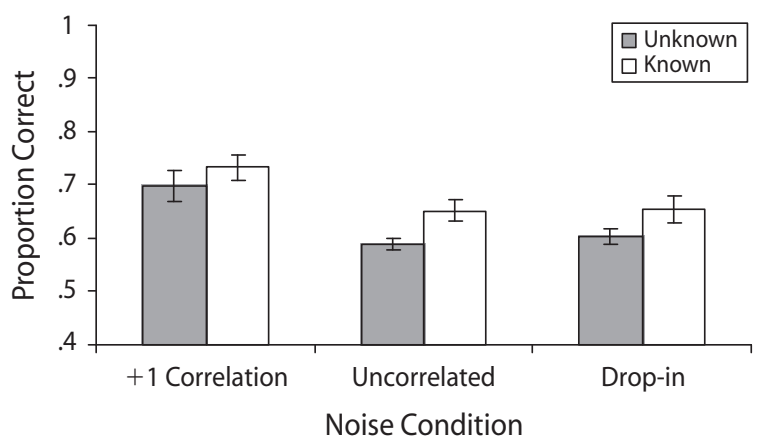

Figure 4. Data from Experiment 2 showing bias-free proportion correct for target detection with positively correlated, uncorrelated, and drop-in noise conditions. Error bars indicate one standard error. 


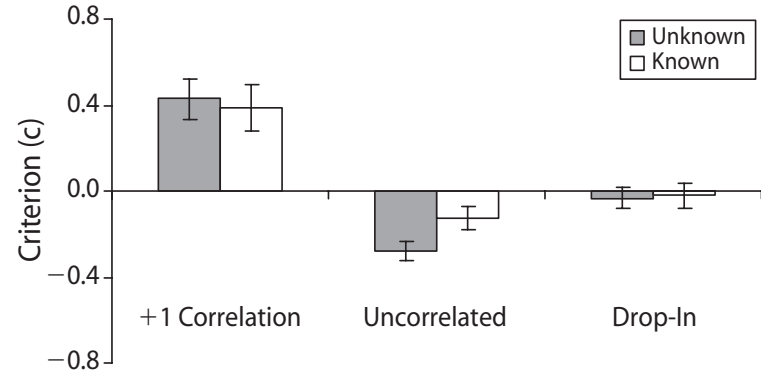

Noise Condition

Figure 5. Data from Experiment 2 showing averaged criterion (c) for target detection in positively correlated, uncorrelated, and drop-in noise conditions. Negative values indicate a bias for "targetpresent" responses and positive values indicate a bias for "targetabsent" responses. One standard error is shown by error bars.

between the drop-in condition and the uncorrelated condition $[t(17)=-0.497, p=.626]$. Performance in both uncorrelated noise and the drop-in condition was affected by knowledge of the target's location $[t(17)=-3.662, p<$ .01 , and $t(17)=-2.050, p=.056$, respectively]. In contrast, sensitivity to targets was unchanged by foreknowledge of the target's location in correlated noise $[t(17)=$ $-1.134, p=.272]$.

The criterion data (shown in Figure 5) were analyzed using a within-subjects ANOVA with target location (known and unknown) and noise (positively correlated, uncorrelated, and drop-in) as factors. There was a reliable effect of noise $[F(2,34)=23.901, p<.01]$, but not of knowledge of target location $[F(1,17)=.727, p=.406]$. The interaction between knowledge of target location and noise condition was not significant $[F(2,34)=2.343, p=.111]$.

With positively correlated noise there was a preference for signal absent responses $[t(17)=4.553, p<.01]$, both when the target location was known and when it was unknown $[t(17)=3.561, p<.01]$. The bias did not change as a function of target foreknowledge. In uncorrelated noise, the bias was for target-present responses, both when the target's location was known $[t(17)=-5.988, p<.01]$ and when it was unknown $[t(17)=-2.240, p<.05]$. This bias was greater when the target's location was unknown $[t(17)=-2.450, p<.05]$. In contrast, there was no bias in either direction in the drop-in conditions, both when the target's location was unknown $[t(17)=-0.769, p=.453]$ and when it was known $[t(17)=-0.277, p=.785]$.

\section{Discussion}

In agreement with the results of Experiment 1, targets were easier to detect when they were presented in bilateral noise that was positively correlated than in noise that was uncorrelated across the hands. In addition, performance in positively correlated noise was also better than that in the drop-in noise condition. This result suggests that participants did capitalize on the initial period of noise during which the correlated and drop-in conditions differed. Although knowing where the target would appear did not change performance when the noise waveforms were positively correlated, it did affect performance in both the uncorrelated and drop-in conditions. It was easier to detect targets in the uncorrelated and drop-in conditions when their locations were known than when they were unknown. Although there was no reliable effect of knowledge of target location in Experiment 1, there was a numeric tendency for better performance when the target location was known than when it was unknown in uncorrelated noise. The results of the present experiment suggest that knowledge of target location may have affected performance with uncorrelated noise in the first experiment.

One account for the results of Experiment 2 is that information from the nontarget hand is filtered out when bilateral patterns are not detected. Therefore, although there is no filtering of inputs when the noise is positively correlated, the lack of any detectable pattern across the hands leads to filtering of "irrelevant inputs" from the nontarget hand. Thus, target detection in the uncorrelated and dropin conditions improves when noise from the nontarget hand can be filtered out. This result leaves the question of why the positive correlation in the drop-in condition was not detected. One possibility is that filtering occurs relatively soon after movement onset and that any subsequent correlation is not picked up. Alternatively, it may be that detection of correlation in kinesthesia is a relatively slow process requiring sampling of a series of signals over time. The window over which this sampling takes place may depend on factors such as the sensitivity to the movement and positions of the fingers. With decreasing sensitivity to stimuli, one may reasonably expect increasingly noisy sensory representations. Central representations of the finger movements will, in turn, be less correlated at each time step than the original stimuli. However, over time these representations will show high degrees of correlation. In the drop-in condition, the onset of the target occurs relatively soon $(200 \mathrm{msec})$ after the noise becomes correlated. This will have led to an effective decrease in correlation between the two hands because of the small sampling time and the presence of the target, making the periods of correlation difficult to detect. It is possible that with longer periods of correlated noise in the drop-in condition, performance would have more closely resembled that found when the noise waveforms were correlated throughout a trial.

\section{EXPERIMENT 3}

In Experiment 3, we investigated whether the filtering of a task-irrelevant hand is set up early in a trial. This investigation was done using a converse condition to the drop-in condition of Experiment 2. Instead of uncorrelated noise with a brief burst of correlated noise (the dropin condition), the noise was positively correlated with a short period of uncorrelated noise around the target period (a "drop-out" condition). The first 1,800 msec of a dropout trial were the same as those of a positively correlated trial. The noise waveforms on the left and right fingers were highly positively correlated. However, $200 \mathrm{msec}$ before and up to $200 \mathrm{msec}$ after the period in which the target could occur, the positive correlation between the 
two hands was removed. The waveform on one of the two fingers (selected at random) was replaced by another waveform that was randomly selected from the base set. Thus, for a period of $1,400 \mathrm{msec}$ (during which the target could occur), the noise movements of the two hands were uncorrelated, and $200 \mathrm{msec}$ after the target interval, the movements of the index fingers once again became positively correlated.

At the start of a given trial, participants may detect that the noise waveform is positively correlated for the two hands. Regardless of whether this detection is implicit or explicit (see Roberts et al., 2005, for evidence of implicit detection of correlation), the target is then more easily discriminated from the noise waveforms than if the noise waveforms had been either bilateral but uncorrelated or on one hand alone. Let us suppose that this improvement occurs because in the context of positively correlated noise, some form of comparison process is undertaken (e.g., subtraction of the inputs of one hand from that of the other). The effect of such a comparison process would be to reduce the noise on the hand on which the target appears. When no correlation is detected, any such comparison process may be stopped and, when the target location is known, the inputs from the nontarget hand may be filtered out. The drop-out condition creates a situation that encourages participants to behave as if the noise waveforms are correlated, but for which there is no advantage in comparing the signals on the two hands. For example, subtracting the signals of one hand from the other will not make the target any easier to detect. Instead, maintaining this comparison process should mean that performance in the drop-out condition is worse than that in the positively correlated condition and that it is unaffected by cues to target location.

\section{Method}

Participants. Eighteen participants, 13 female and 5 male, participated in this experiment after giving informed consent. Their ages ranged from 19 to 35 years $(M=22, S D= \pm 4.3)$. Fourteen participants were right handed, 4 were left handed for handwriting, and all were paid for the time spent in the experiment. These participants were not informed of the purpose of this study.

Procedure. The procedure used in this experiment was the same as that used in Experiment 1 .

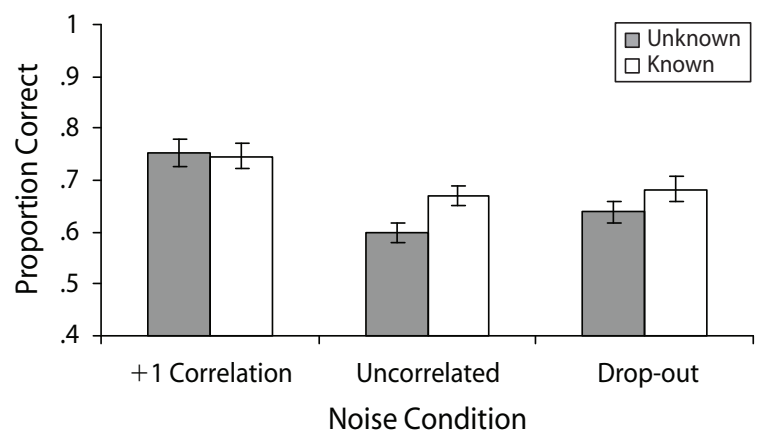

Figure 6. Data from Experiment 3 showing bias-free proportion correct for target detection with positively correlated, uncorrelated, and drop-out noise conditions.

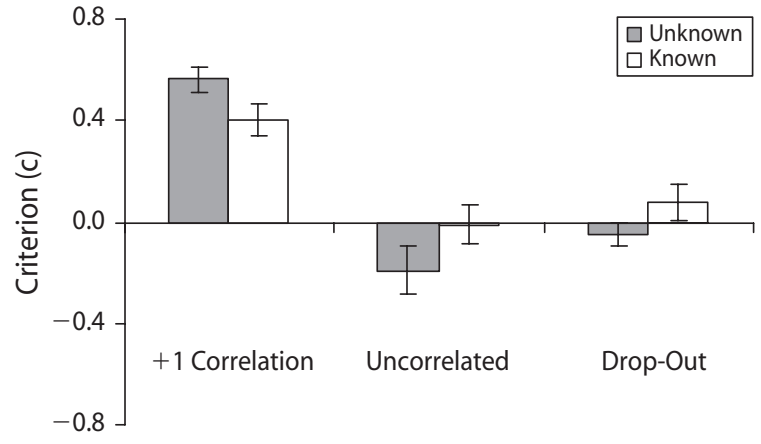

Noise Condition

Figure 7. Data from Experiment 3 showing averaged criterion (c) for target detection in positively correlated, uncorrelated, and drop-out noise conditions. Negative values indicate a bias for "target-present" responses, and positive values indicate a bias for "target-absent" responses.

Design. The noise waveforms could be positively correlated, uncorrelated, or they could drop out of correlation. The participants were not informed of the different noise conditions.

Each participant was tested on a total of 360 trials that were run in three blocks of 90,90 , and 180 trials. In one block of 90 trials, the target would only occur on the left, and in the other block of 90 trials, the target would only occur on the right. In both of these blocks, participants were told in advance on which hand the target would appear (known condition). In contrast, targets occurring during the block of 180 trials could appear on the left or right hand, and participants had no prior knowledge of target position (unknown condition). In all blocks, half of the trials contained targets, and the other half did not. The order of the location conditions (known vs. unknown) was balanced across the participants. The trials for the different noise conditions were interleaved within each block.

\section{Results}

The proportion of correct responses averaged across participants is shown in Figure 6. There was no main effect of the order in which the different location conditions (known vs. unknown) were run. In addition, there were no reliable interactions involving location order. Consequently, all of the following analyses were conducted with performance averaged across order. The data were analyzed using a within-subjects ANOVA with target location (known and unknown) and noise (positively correlated, uncorrelated, and drop-out) as factors.

There was a reliable main effect of noise $[F(2,34)=$ 23.187, $p<.01$ ], but no significant effect of knowledge of target location on sensitivity $[F(2,17)=3.161, p=$ .093]. There was, however, an interaction between noise and target location $[F(2,34)=4.705, p<.05]$. A separate within-subjects ANOVA that compared performance in just the uncorrelated and drop-out conditions revealed a significant effect of target location $[F(1,17)=7.931, p<$ $.05]$ but not of noise $[F(1,17)=2.725, p=.117]$, and no interaction between these factors $[F(1,17)=1.275, p=$ .275]. With foreknowledge of target location more targets were detected in uncorrelated noise $[t(17)=-3.549, p<$ .01 ; one-tailed]. This difference approached significance in the drop-out condition [t(17) $=-1.632, p=.061]$. Thus, the drop-out condition did not differ from the uncor- 
related condition, and it was equally affected by knowledge of target location. There was no effect of foreknowledge of the target location with correlated noise $[t(17)=0.172$, $p=.866]$.

The criterion data, shown in Figure 7, were analyzed using a within-subjects ANOVA with target location (known and unknown) and noise (positively correlated, uncorrelated, and drop-out) as factors. There was a reliable effect of noise $[F(2,34)=35.736, p<.01]$, but not of knowledge of target location $[F(1,17)=1.214, p=.286]$. There was a significant interaction between knowledge of target location and noise condition $[F(2,34)=8.901, p<.01]$. A separate within-subjects ANOVA that compared performance in just the uncorrelated and drop-out conditions revealed a significant effect of target location $[F(1,17)=8.396, p<$ $.05]$ and of noise $[F(1,17)=5.011, p<.05]$, but no interaction between them $[F(1,17)=.428, p=.522]$. There was a reliable effect of foreknowledge of the target location with correlated noise $[t(17)=2.455, p<.05]$.

There was a tendency to respond "target-absent" when the noise waveforms were in positively correlated noise, both when the target location was known $[t(17)=6.436$, $p<.01]$ and unknown $[t(17)=10.818, p<.01]$. This tendency decreased with foreknowledge of target location $[t(17)=2.455, p<.05]$.

\section{Discussion}

The results again confirm that discrimination was better when there was correlated noise on the two hands than when the noise was uncorrelated. In this experiment, as in Experiments 1 and 2, there was an advantage to knowing where the target could occur when the noise was uncorrelated bilaterally. This advantage suggests that given foreknowledge of the target location, participants can effectively ignore uncorrelated noise that occurs on the nontarget hand across the trial. Performance in the drop-out condition was similar to that in the uncorrelated noise condition, also improving with knowledge of target location. It is interesting that performance in the drop-out condition was affected by foreknowledge. If participants compared the noise on the two hands during the drop-out period, regardless of whether they had foreknowledge about the target location, then performance in the drop-out condition could have been worse than when there was foreknowledge of the target's location in the uncorrelated condition.

Two alternative ways of interpreting these results are that (1) the use of correlation occurs independently of the focus of attention or (2) there is rapid switching of attention between the inputs presented to the two hands. In the first case, processes responsible for the detection and use of correlation may be independent of how attention is distributed. Participants may make use of correlation but also filter the inputs from one hand. When the noise is correlated, the bilateral comparison process may contribute the most to performance. However, when these cues are no longer available, as in the drop-out condition, performance will be primarily determined by the degree to which the noise from the nontarget hand can be filtered out. This account is in agreement with previous findings that temporally lagged but positively correlated noise leads to improved target discrimination. This improvement is observed even though such lagged patterns cannot be explicitly discriminated from noise that is uncorrelated (Roberts et al., 2005). Against this interpretation, there is no evidence of filtering when the noise is positively correlated. If independent filtering and comparison processes were both contributing to performance, then target detection in correlated noise should have shown effects of knowledge of target location.

An alternative account for the results of Experiment 3 is that participants may be sensitive to the change in correlation during the drop-out period. When the target's location is known, they rapidly switch attention in the critical $200 \mathrm{msec}$ prior to the target occurring, minimizing the cost from the uncorrelated noise. This account suggests that the filtering of task-irrelevant inputs may not be set up early in the trial. The processing of inputs from the two hands may instead change in a flexible manner, responding to any changes in bilateral patterns.

\section{GENERAL DISCUSSION}

Past research has shown that patterns of coordination in the sensory inputs across the two hands are not only detected but also shape the way in which those inputs are perceived (Roberts et al., 2005). The present set of experiments extended these findings by investigating how perception with bilateral inputs might differ from that with unilateral inputs, and whether the effects of bilateral patterns in noise are modulated by the deployment of attention.

In agreement with previous findings, it was easier to detect the presence of a discrete, unilateral kinesthetic target when it was accompanied by ongoing, bilateral kinesthetic noise that was correlated than with noise that was uncorrelated. This difference in performance was not simply due to increased variation in uncorrelated trials. Positively correlated noise led to an increase in detection in comparison with a baseline condition in which the target was masked by noise occurring on one hand alone. This result is consistent with the bilateral correspondence between the hands affecting the way in which we interpret the signals from each hand, leading to an improvement in the ability to separate the discrete target from the ongoing noise stimuli.

Improved performance with positively correlated noise was consistently unaffected by whether both hands were task relevant or not. This result was in contrast to performance in the uncorrelated condition in which knowing where the target would occur provided a distinct advantage. The benefits of positive correlation were eliminated when the noise was uncorrelated in the period running up to the target, but were positively correlated during the period in which the target could occur (the drop-in condition, in Experiment 2). Performance in this drop-in condition did not differ from the condition with uncorrelated noise on the hands. In both conditions, sensitivity increased when participants had foreknowledge of the target's location. Behavior was also measured in a converse condition in which an initial period of correlated noise was followed by $200 \mathrm{msec}$ of uncorrelated noise prior to 
the target (the drop-out condition). In this drop-out condition, performance was still affected by knowledge of target location. Knowing where the target could occur produced similar levels of performance whether the bilateral noise was either entirely uncorrelated or correlated with a burst of uncorrelated noise during the target period.

Although the effects of noise (positively correlated and uncorrelated) and foreknowledge on target detection were consistent across all three experiments, their impact on the biases was less clear cut. The tendency to favor targetabsent responses with positively correlated noise both did (Experiment 3) and did not (Experiments 1 and 2) vary with foreknowledge of target location. In contrast, with uncorrelated noise, there was always a difference in the criterion adopted when the target's location was unknown as opposed to when it was known, but there was no systematic preference for one response over another. Biases were small or absent with all other noise conditions. This variability in response preference makes it difficult to posit an explanation for biases shown in each individual experiment. There is no obvious reason for the biases found, since participants were informed that there were equal numbers of target-present and target-absent trials. Moreover, there was no reward for one response over another.

On the whole, the sensitivity results show that there is filtering of kinesthetic inputs from a hand that is task irrelevant. However, this use of filtering changes depending on the level of correspondence between the inputs on the hands. When the hands experience highly similar stimuli, there is little filtering of inputs from a nontarget hand. In contrast, when the inputs on one hand appear unrelated to those on the other hand, inputs from the nontarget hand are filtered, resulting in better detection of the target. The most parsimonious account of the drop-in and drop-out results is that rather than being rigidly engaged at the beginning of a trial, filtering appears to operate in a flexible manner, responding to ongoing changes in correlation. When a previously high level of correlation is purposely reduced before the onset of a target, irrelevant inputs are then filtered and performance varies with foreknowledge of target location. Conversely, detection of correlation in previously uncorrelated inputs should give rise to less filtering and an increase in sensitivity to discrete stimuli appearing on only one of the hands. Although this last prediction was not found with uncorrelated noise containing a brief period of correlated noise (Experiment 2), it is possible that this may have been due to the use of bursts of correlation that were too brief to be detected. The effect of a decrease in correlation but not an increase in correlation on target detection in the present experiments may reflect differential sensitivity to the changes in correlation, depending on the direction of change.

Evidence that sensitivity to changes in correlation may depend on the direction of the change are found in audition. Indeed, as in kinesthesia, targets presented to one ear have been shown to be affected by correlation in noise presented to both ears. These findings come in part from experiments in which participants are asked to lateralize (Egan \& Benson, 1966; Robinson \& Egan, 1974) or detect the presence of (Blodgett, Jeffress, \& Whitworth, 1962; Hirsh, 1948; Hirsh \& Burgeat, 1958; Licklider, 1948; Weston \& Miller, 1965) a pure tone presented to one ear (monaural target) in the presence of broadband noise presented to both ears (binaural noise). Generally, such experiments show that the target intensity required to detect a target (at a set level of performance) can be lowered under certain interaural conditions - for example, when the noise is binaural and positively correlated in comparison with when it is monaural (Blodgett et al., 1962; Green, 1966; Hirsh \& Burgeat, 1958; Weston \& Miller, 1965; Wilbanks \& Whitmore, 1968). This difference in thresholds with the addition of noise to a nontarget ear is known as the masking level difference (MLD). MLDs have also been found when the thresholds for tones detected in positively correlated noise are compared with those measured for detection in noise that has a zero or low correlation across the ears (corresponding to the uncorrelated conditions in the present experiments) (Egan \& Benson, 1966; Robinson \& Egan, 1974; Weston \& Miller, 1965; Wilbanks \& Whitmore, 1968). Moreover, research has shown that there is better detection of decreases than increases in correlation (Boehnke, Hall, \& Marquardt, 2002; Culling, Colburn, \& Spurchise, 2001; Trittipoe \& Pollock, 1959), a result similar in kind to what we presently report (more rapid reaction to a decrease in correlation than to the emergence of correlation). This difference could be because in order to detect a temporal correlation, signals must, by definition, occur over time, making the detection of correlation slow. In contrast, the jump from correlated to noncorrelated input may be detected from the first disparate signal.

A further interesting comparison between this auditory phenomenon and our findings in kinesthesia is that the effects of noise on auditory target detection appear immune to uncertainty about which ear will receive the target. Egan and Benson (1966) compared target detection when the location of the target was restricted to one side throughout a block with that when the target could occur equally often on either ear. Detection in both positively correlated and uncorrelated noise showed very little change with certainty of the target's location. Even though participants have foreknowledge of the target's location, it appears that they continue to process and use the information from both ears regardless of the level of correlation across the ears.

It is unlikely that processes involved in the handling of multiple auditory stimuli would be those responsible for the processing of multiple touch inputs, since there are fundamental differences between the different sensory systems. For instance, at a very basic level, the set of sensory organs in audition have a fixed relative location. We cannot change the location of one ear without affecting the position of the other. Furthermore, we are able to hear events that are both distant and close to our bodies. By contrast, we can only feel objects within our reach, which may be somewhat extended through the use of tools (Yamamoto \& Kitazawa, 2001; Yamamoto, Moizumi, \& Kitazawa, 2005). Furthermore, our hands and fingers can adopt a large number of spatial configurations and show idiosyncratic patterns of 
movement when used in a sensory capacity (Lederman \& Klatzky, 1987). Such differences between the senses may fundamentally affect not only the sorts of patterns that one would expect across multiple sensory streams, but also the way in which those patterns might affect perception. For instance, a pair of correlated sounds may be localized at a point midway between their true sources. This localization, varying as a function of interaural differences in amplitude or phase, facilitates the perception of a single source for the correlated sounds. There is no evidence to suggest that similar changes should be expected in kinesthesia. However, observe that the different sensory systems adopt a similar general approach to the problem of parsing inputs from multiple sources - that is, detecting and using coordinated changes over time to create a stable and coherent perception of moving objects or an event. Furthermore, the differences between the modalities are informative about the underlying processing mechanisms that govern perception in each modality.

In the present article, the signals are referred to as kinesthetic. This term has been commonly used to refer to the perceptual experience of limb states, broadly encompassing the sense of position, movement and loading of the limbs. These sensations are mediated through the skin, muscle and joint receptors (Gandevia, Hall, McCloskey, \& Potter, 1983; Gandevia \& McCloskey, 1976; Refshauge, Kilbreath, \& Gandevia, 1998). Throughout this article, we have discussed stimulation in terms of changes in position over time. It is possible that participants rely on position signals to accomplish the task, detecting and making use of any correlations in these signals. However, it is also possible that signals indicating the rate of change of position (velocity) were used to perform the task. This is particularly relevant to the present task in which the fingers were stimulated, since there is evidence that sensory receptors in the muscles of the fingers are specifically tuned to detecting changes in velocity. Muscle spindles have been shown to respond not only to length but also to velocity (Edin \& Vallbo, 1990; Houk, Rymer, \& Crago, 1981). Furthermore, this sensitivity to velocity is also evident in area $3 \mathrm{a}$ in the primary somatosensory cortex, an area receiving most of its peripheral inputs from muscle spindles signaling muscle stretch information (Jones \& Porter, 1980) and thought to play a role in integrating information within and between different body part representations (Huffman \& Krubitzer, 2001). Using single unit recordings, Wise and Tanji (1981) examined how neurons in area 3a responded to ramp displacements of the foot in unanesthetized monkeys. They found that peak activity in area 3a was highly correlated with the velocity of the imposed movement. Consider also that this otherwise kinesthetic task may involve the use of tactile cues. Acceleration is directly coupled to the actuator forces, and these cues would translate into pressure on the fingers. Thus, pressure variation may also have provided a potential means for tactile input to contribute to performance in this task.

We have shown that in kinesthesia, the effects of noise on the detection of unilateral targets is reduced when correlated noise is present to both hands in comparison with when the noise on each hand is unrelated or when only unilateral noise occurs. Moreover, noise signals from a task-irrelevant hand are filtered only in those conditions in which there is a low correspondence between the hands. This finding suggests a system in which the inputs for both hands are continually and possibly preattentively compared in order to take advantage of matching input.

\section{AUTHOR NOTE}

This article forms part of the $\mathrm{PhD}$ thesis of R.D.R. and was supported by funding from the Economic and Social Sciences Research Council (ESRC), the Medical Research Council (MRC-UK), and the European Union. Correspondence concerning this article should be addressed to R. D. Roberts, Behavioural Brain Sciences Centre, School of Psychology, University of Birmingham, Birmingham B15 2TT, England (e-mail: r.roberts@bham.ac.uk).

\section{REFERENCES}

Bingham, G. P., Schmidt, R. C., \& ZaAL, F. T. J. M. (1999). Visual perception of the relative phasing of human limb movements. Perception \& Psychophysics, 61, 246-258.

Bingham, G. P., ZaAl, F. T. J. M., Shull, J. A., \& Collins, D. R. (2001). The effect of frequency on the visual perception of relative phase and phase variability of two oscillating objects. Experimental Brain Research, 136, 543-552.

Blodgett, H. C., Jeffress, L. A., \& Whitworth, R. H. (1962). Effect of noise at one ear on the masked threshold for tone at the other. Journal of the Acoustical Society of America, 34, 979-981.

Boehnke, S. E., Hall, S. E., \& Marquardt, T. (2002). Detection of static and dynamic changes in interaural correlation. Journal of the Acoustical Society of America, 112, 1617-1626.

Culling, J. F., Colburn, H. S., \& Spurchise, M. (2001). Interaural correlation sensitivity. Journal of the Acoustical Society of America, 110, 1020-1029.

Edin, B. B., \& Vallbo, A. B. (1990). Dynamic response of human muscle spindle afferents to stretch. Journal of Neurophysiology, 63, 1297-1306.

EgAn, J. P., \& BENSON, W. (1966). Lateralization of a weak signal presented with correlated and with uncorrelated noise. Journal of the Acoustical Society of America, 40, 20-26.

FITTS, P. M. (1992). The information capacity of the human motor system in controlling the amplitude of movement. Journal of Experimental Psychology: General, 121, 262-269.

Gandevia, S. C., Hall, L. A., McCloskey, D. I., \& Potter, E. K. (1983). Proprioceptive sensation at the terminal joint of the middle finger. Journal of Physiology, 335, 507-517.

Gandevia, S. C., \& McCloskey, D. I. (1976). Joint sense, muscle sense, and their combination as position sense, measured at the distal interphalangeal joint of the middle finger. Journal of Physiology, 260, 387-407.

GREEN, D. M. (1966). Interaural phase effects in the masking of signals of different durations. Journal of the Acoustical Society of America, 39, 720-724.

Hirsh, I. J. (1948). The influence of interaural phase on interaural summation and inhibition. Journal of the Acoustical Society of America, 20, 536-544.

Hirsh, I. J., \& BurgeAt, M. (1958). Binaural effects in remote masking. Journal of the Acoustical Society of America, 30, 827-832.

Houk, J. C., Rymer, W. Z., \& Crago, P. E. (1981). Dependence of dynamic response of spindle receptors on muscle length and velocity. Journal of Neurophysiology, 46, 143-166.

Huffman, K. J., \& Krubitzer, L. (2001). Area 3a: Topographic organization and cortical connections in marmoset monkeys. Cerebral Cortex, 11, 849-867.

Jones, E. G., \& Porter, R. (1980). What is area 3a? Brain Research, 203, 1-43.

Kelso, J. A. S. (1984). Phase transitions and critical behavior in human bimanual coordination. American Journal of Physiology, 246, R1000-R1004.

Kelso, J. A. S., Putnam, C. A., \& Goodman, D. (1983). On the spacetime structure of human interlimb coordination. Quarterly Journal of Experimental Psychology, 35A, 347-375. 
Kelso, J. A. S., Southard, D. L., \& Goodman, D. (1979). On the coordination of two-handed movements. Journal of Experimental Psychology: Human Perception \& Performance, 5, 229-238.

Kitada, R., Kochiyama, T., Нashimoto, T., Naito, E., \& MaTSUMURA, M. (2003). Moving tactile stimuli of fingers are integrated in the intraparietal and inferior parietal cortices. NeuroReport, 14, 719-724.

Lederman, S. J., \& KLATZKy, R. L. (1987). Hand movements: A window into haptic object recognition. Cognitive Psychology, 19, 342-368.

LICKLIDER, J. C. R. (1948). The influence of interaural phase relations upon the masking of speech by white noise. Journal of the Acoustical Society of America, 20, 150-158.

Macmillan, N. A., \& Creelman, C. D. (1991). Detection theory: A user's guide (1st ed.). New York: Cambridge University Press.

Perrig, S., Kazennikov, O., \& Wiesendanger, M. (1999). Time structure of a goal-directed bimanual skill and its dependence on task constraints. Behavioural Brain Research, 103, 95-104.

Refshauge, K. M., Kilbreath, S. L., \& Gandevia, S. C. (1998). Movement detection at the distal joint of the human thumb and fingers. Experimental Brain Research, 122, 85-92.

Ridderikhoff, A., Peper, C. L., \& Beek, P. J. (2005). Unraveling interlimb interactions underlying bimanual coordination. Journal of Neurophysiology, 94, 3112-3125.

Ridderikhoff, A., PePer, C. L., \& Beek, P. J. (2006). Bilateral phase entrainment by movement-elicited afference contributes equally to the stability of in-phase and antiphase coordination. Neuroscience Letters, 399, 71-75.

Roberts, R. D., Humphreys, G. W., \& Wing, A. M. (2005). Temporal constraints on interactions across kinaesthetic channels. Experimental Brain Research, 164, 529-540.

Robinson, D. E., \& Egan, J. P. (1974). Lateralization of an auditory signal in correlated noise and in uncorrelated noise as a function of signal frequency. Perception \& Psychophysics, 15, 281-284.

Serrien, D. J., Li, Y., Steyvers, M., Debaere, F., \& Swinnen, S. P. (2001). Proprioceptive regulation of interlimb behavior: Interference between passive movement and active coordination dynamics. Experimental Brain Research, 140, 411-419.

Serrien, D. J., \& Wiesendanger, M. (2001a). Bimanual organization of manipulative forces: Evidence from erroneous feedforward programming of precision grip. European Journal of Neuroscience, 13, $1825-1832$.

SERrien, D. J., \& Wiesendanger, M. (2001b). A higher-order mechanism overrules the automatic grip-load force constraint during bimanual asymmetrical movements. Behavioural Brain Research, 118, 153-160.

Trittipoe, W., \& Pollock, I. (1959). Binaural listening and interaural noise correlation. Journal of the Acoustical Society of America, 31, 127.

Weston, P. B., \& MilleR, J. D. (1965). Use of noise to eliminate one ear from masking experiments. Journal of the Acoustical Society of America, 37, 638-646.

Wilbanks, W. A., \& Whitmore, J. K. (1968). Detection of monaural signals as a function of interaural noise correlation and signal frequency. Journal of the Acoustical Society of America, 43, 785-797.

Wilson, A. D., Bingham, G. P., \& Craig, J. C. (2003). Proprioceptive perception of phase variability. Journal of Experimental Psychology: Human Perception \& Performance, 29, 1179-1190.

Wise, S. P., \& TANJI, J. (1981). Neuronal responses in sensorimotor cortex to ramp displacements and maintained positions imposed on hindlimb of the unanesthetized monkey. Journal of Neurophysiology, $45,482-500$.

Yamamoto, S., \& Kitazawa, S. (2001). Sensation at the tips of invisible tools. Nature Neuroscience, 4, 979-980.

Yamamoto, S., Moizumi, S., \& Kitazawa, S. (2005). Referral of tactile sensation to the tips of L-shaped sticks. Journal of Neurophysiology, 93, 2856-2863.

Yamanishi, J., Kawato, M., \& Suzuki, R. (1980). Two coupled oscillators as a model for the coordinated finger tapping by both hands. Biological Cybernetics, 37, 219-225.

ZaAl, F. T. J. M., Bingham, G. P., \& Schmidt, R. C. (2000). Visual perception of mean relative phase and phase variability. Journal of Experimental Psychology: Human Perception \& Performance, 26, 1209-1220.

(Manuscript received June 5, 2006;

revision accepted for publication June 22, 2007) 